\title{
Progression of Type-2 Diabetes Mellitus Associated with Pulmonary Tuberculosis Sequelae in a 60 Year Old Hypertensive Patient: A Multifactorial Case Report with Literature Review
}

\author{
Kiran Kumar Rathinam and Sarvesh Sabarathinam* \\ Department of Pharmacy Practice, SRM College of Pharmacy, SRM IST, Tamil Nadu, \\ India \\ *Corresponding Author: Sarvesh Sabarathinam, Department of Pharmacy Practice, \\ SRM College of Pharmacy, SRM IST, Tamil Nadu, India.
}

Received: August 29, 2020

Published: September 12, 2020

(C) All rights are reserved by Kiran Kumar

Rathinam and Sarvesh Sabarathinam.

\begin{abstract}
Here we discuss a 60year old patient with pulmonary tuberculosis sequelae followed by uncontrolled diabetes mellitus with complicated conditions. Insulin resistance is one of the main factors which plays a significant role in glucose homeostasis and energy metabolism. Non-adherent to medication and insulin resistance are the primary factors are the causes of diabetes complication such as microvascular and macrovascular complications. Glycated haemoglobin plays a significant role in the identification of drug sensitivity and determination of insulin insufficiency. This case describes the multifactorial complications due to uncontrolled diabetes; the need for screening for $\mathrm{HbA1c}$ is recommended in the high-risk group.
\end{abstract}

Keywords: Diabetes Mellitus; Tuberculosis; Anemia; Hypertension; Oxidative Stress

\section{Introduction}

Diabetes is a metabolic disorder characterized by hyperglycemia due to impaired insulin secretion and insulin deficiency. According to 2017's International diabetes Federation data, 4 million deaths occurred due to diabetes mellitus [1]. Insulin resistance or Deficient insulin production by the pancreas is one of the significant causes, which leads to progression of hyperglycemia. Insulin resistance is one of the primary cause for metabolic syndrome, endothelial dysfunction and elevated renin-angiotensin-aldosterone system [2]. The coexistence of diabetes and hypertension leads to the development of cardiovascular complications and renal diseases. Meanwhile, Renal dysfunction and insufficient production of erythropoietin hormone paves the way for development of Anemia in the early stages of kidney diseases $[3,4]$. Poor glycemic control and immune compromised in diabetic patient have higher incidence of occurrence of TB [5]. The recent clinical studies reveal that Prostatomegaly associated with the pathogenesis of diabetic and obese patients [6]. In this case report, we present a multifacto- rial comorbidities emerging due to the progression of type- 2 diabetes mellitus associated with pulmonary tuberculosis sequelae in a 60 year old hypertensive patient.

\section{Case Report}

A 60 years old male patient admitted in General medicine department with the chief complaints of dyspnea on exertion (grade 3 ), nausea and loose stools, three episodes/day for the past 10 days, urinary retention. The patient had a past medical history of type-2 diabetes mellitus for 14 years, systemic hypertension for the past 10 years and had pulmonary tuberculosis 3 years back and dilated cardiomyopathy for the past 2 years. The patient had a past medication history as follows: Tab. Metformin $500 \mathrm{mg}$, Tab. Trimetazidine $35 \mathrm{mg}$, Tab. Pantoprazole $40 \mathrm{mg}$, Tab. Furosemide 20 $\mathrm{mg}+$ spironolactone $100 \mathrm{mg}$, Tab. Atorvastatin $20 \mathrm{mg}$. The patient had completed first line antitubercular regimen treatment. The patient is alcoholic and chain smoker for the past 15 years. Upon admission, the patient was afebrile and conscious oriented. Physi- 
cal examination was performed and reveals tracheal deviation over the right side of the chest, wheeze over the chest, increased vocal fremitus, and vocal resonance in the right side of the chest, loss of skin turgor. The haematological parameters assessed, and abnormal values are as follows: haemoglobin- $9.2 \mathrm{~g} / \mathrm{dl}, \mathrm{RBC}-3.5$ million cells/cu.mm, PCV- 29\%, MCV- 81 f L, ESR- 15mm/Hr. The peripheral blood smear was examined, found with moderate normocytic anaemia, neutrophilic preponderance. The liver function test shows: Total bilirubin- $1.5 \mathrm{mg} / \mathrm{dl}$, direct bilirubin- $0.7 \mathrm{mg} / \mathrm{dl}$, SGOT- 3050 U/L, SGPT- 1835 U/L, ALP- 283 U/L, Albumin- 3.2 g/ $\mathrm{dl}$, globulin-4.1g/dl. The renal function test shows: serum creatinine- $1.5 \mathrm{mg} / \mathrm{dl}$, serum urea- $63 \mathrm{mg} / \mathrm{dl}$, uric acid- $9.5 \mathrm{mg} / \mathrm{dl}$. The blood glucose levels monitored, and FBS, RBS, PPBS values were found to be elevated. Especially haemoglobin A1C was found to be $11 \%$. Abdomen Ultrasonography performed, and it reveals fatty liver, mild ascites, right minimal pleural effusion, grade- 2 prostatomegaly with median lobe projecting into the bladder, bilateral raised renal cortical echoes. Chest radiography was performed, and it shows cardiomegaly, right destroyed lung. Electrocardiogram performed, and it reveals poor R- wave progression, ST inversion in V5-V6. Electrocardiography shows left ventricular ejection fraction- $36 \%$, grade 2 diastolic dysfunction, mild mitral regurgitation, trivial tricuspid regurgitation, dilated left atrium, mild pulmonary hypertension- $35 \mathrm{mmHg}$. Computed tomography scan of chest region performed, it shows destroyed upper lobe of the right lung with a large cavity, consolidation over the right middle lobe of left inguinal, bilateral pleural effusion (more over right side compared to the left side). As per subjective and objective evidence, the patient diagnosed with type- 2 diabetes mellitus, hypertension, old pulmonary tuberculosis sequelae, dilated cardiomyopathy, alcoholic liver disease, acute kidney injury, anaemia, benign prostate hyperplasia and atopic dermatitis.

\section{Discussion and Literature Review}

In type 2 diabetes patients, hypertension aggravates the risk of cardiovascular disorders [7]. The synchronicity of diabetes mel- litus and systemic hypertension identified more in the untreated group, paves the onset of chronic kidney diseases. Pathologically the prolonged biological stress over the endoplasmic reticulum potentiates the nephrotic cellular injury [8]. The underlying association between diabetes and hypertension includes insulin resistance in Nitric oxide pathway, changes in renin-angiotensin-aldosterone pathway due to hyperglycemia, the hyperinsulinemia effects over the smooth muscles, which results in tissue damage [9]. The poor glycemic control in diabetes patients affects the cell-mediated cytokine immune responses in pulmonary TB patients, as well as Anti Tubercular regimens itself, aggravates the blood glucose level. By initiating the immune therapy in diabetes patients can reduce the onset of TB. Pathological exposures to hyperinsulinemia induce the left ventricular dysfunction, decreased LV ejection fraction, left ventricular hypertrophy which leads to diabetic cardiomyopathy. The triggering mechanism associated with diabetic cardiomyopathy includes abnormalities in insulin signalling, dysfunction of autonomic nervous system and inflammation of myocardial cells [10]. Few clinical studies concluded that the association between obesity and increased blood glucose with benign prostatic hyperplasia by promoting metabolic disturbances [11]. Consequently, uncontrolled hyperglycemia will lead to vascular complications and prostate enlargement [12]. The pathogenesis of type 2 diabetes mellitus in acute kidney failure is multifactorial. Around $40 \%$ of diabetic patients develop kidney disease due to glomerular hyperfiltration, Glomerular hypertrophy, progressive albuminuria and glomerulosclerosis caused by metabolic imbalance [13]. Progression of anaemia in CKD due to renal damage caused by elevated proinflammatory mediators resulting in erythropoietin deficiency [14]. Additionally, insulin deficiency affects the equilibrium between differentiation of keratinocytes and its proliferation which leads to topical dermatitis or psoriasis [15]. Due to diabetes-induced cardiovascular and renal complications, improper filtration of urea in the kidney leads to sedimentation in the subcutaneous tissues causes xerosis and itchy skin [16]. Figure A shows an outline of the pathogenesis of diabetes mellitus inducing multiple comorbidities.

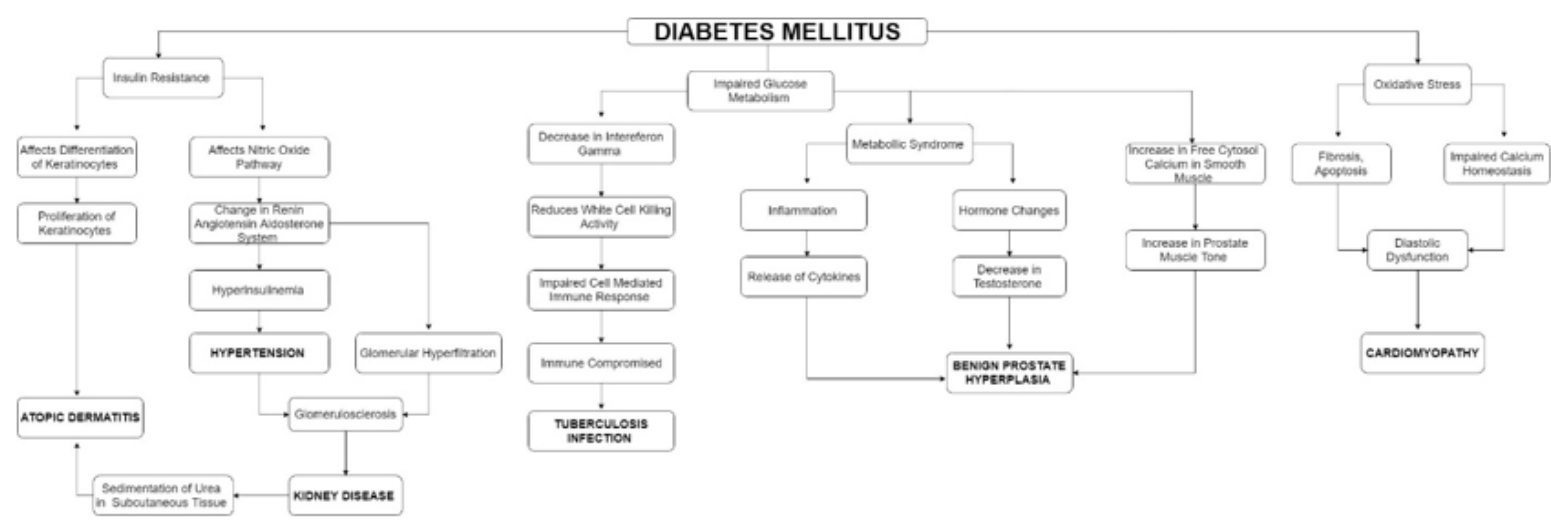

Figure A

Citation: Kiran Kumar Rathinam and Sarvesh Sabarathinam. "Progression of Type-2 Diabetes Mellitus Associated with Pulmonary Tuberculosis Sequelae in a 60 Year Old Hypertensive Patient: A Multifactorial Case Report with Literature Review”. Acta Scientific Pharmaceutical Sciences 4.10 (2020): 12-14. 


\section{Conclusion}

Development of co-morbid conditions mentioned above in diabetes patients requires exceptional health care towards their management. We conclude that progression of untreated diabetes strengthens co-morbid diseases. Clinicians can alter the therapy based on the patient's glycemic control as well as substantiate whether the medications are sensitive to patient conditions. Hence this case stands as an exemplary report for emergence of a multifactorial comorbidities due to the progression of type- 2 diabetes mellitus.

\section{Bibliography}

1. World Health Organization. Diabetes mellitus.

2. Gurushankar Govindarajan., et al. "Hypertension and Diabetes Mellitus”. European Cardiovascular Disease 2.1 (2006): 21-24.

3. Sowers J R. "Treatment of hypertension in patients with diabetes". Archives of Internal Medicine 164.17 (2004): 1850-1857.

4. El-Atat F., et al. "Diabetes, hypertension, and cardiovascular derangements: pathophysiology and management". Current Hypertension Reports 6.3 (2004): 215-223.

5. Leung CC., et al. "Diabetic control and risk of tuberculosis: a cohort study". American Journal of Epidemiology 12.12 (2008): 1486-1494.

6. Sarma AV., et al. "Associations between diabetes and clinical markers of benign prostatic hyperplasia among communitydwelling Black and White men". Diabetes Care 31.3 (2008): 476-482.

7. Fa'iza Abdullah., et al. "Prevalence of Hypertension and Glycaemic Control in Adult Type-2 Diabetes Patients: A Preliminary Retrospective Cohort Study in Kuantan, Pahang, Malaysia”. International Medical Journal Malaysia 16 (2017): 115-121.

8. Wang Z., et al. "Synergistic Interaction of Hypertension and Diabetes in Promoting Kidney Injury and the Role of Endoplasmic Reticulum Stress". Hypertension 69.5 (2017): 879-891.

9. Ferrannini E and Cushman WC. "Diabetes and hypertension: the bad companions". Lancet 380.9841 (2012): 601-610.

10. Jia G., et al. "Insulin resistance and hyperinsulinaemia in diabetic cardiomyopathy". Nature Reviews Endocrinology 12.3 (2015): 144-153.
11. I Kellogg Parsons., et al. "Metabolic Factors Associated with Benign Prostatic Hyperplasia". The Journal of Clinical Endocrinology and Metabolism 91.7 (2006): 2562-2568.

12. Xiaobing Qu., et al. "Prostate volume correlates with diabetes in elderly benign prostatic hyperplasia patient". International Urology and Nephrology 46 (2014): 499.

13. Radica Z Alicic., et al. "Diabetic Kidney Disease". CJASN 12.12 (2017): 2032-2045.

14. Ryu SR., et al. "The Prevalence and Management of Anemia in Chronic Kidney Disease Patients: Result from the Korean Cohort Study for Outcomes in Patients With Chronic Kidney Disease (KNOW-CKD)". Journal of Korean Medical Science 32.2 (2016): 249-256.

15. Napolitano., et al. "Insulin Resistance and Skin Diseases". The Scientific World Journal (2015): 1-11.

16. Neilly JB., et al. "Pruritus in diabetes mellitus: investigation of prevalence and correlation with diabetes control". Diabetes Care 9.3 (1986): 273-275.

\section{Assets from publication with us}

- Prompt Acknowledgement after receiving the article

- Thorough Double blinded peer review

- Rapid Publication

- Issue of Publication Certificate

- High visibility of your Published work

Website: $\underline{w w w}$.actascientific.com/

Submit Article: www.actascientific.com/submission.php

Email us: editor@actascientific.com

Contact us: +919182824667

Citation: Kiran Kumar Rathinam and Sarvesh Sabarathinam. "Progression of Type-2 Diabetes Mellitus Associated with Pulmonary Tuberculosis Sequelae in a 60 Year Old Hypertensive Patient: A Multifactorial Case Report with Literature Review". Acta Scientific Pharmaceutical Sciences 4.10 (2020): $12-14$. 